\title{
O uso do campo MARC 9XX para controle bibliográfico institucional
}

\section{Zita Prates de Oliveira}

Bibliotecária de sistemas, Centro de Processamento de Dados da UFRGS, doutora em ciências da comunicação (USP).

E-mail:zita@cpd.ufrgs.br.

\section{Caterina Groposo Pavão}

Bibliotecária de sistemas, Centro de Processamento de Dados da UFRGS, bacharel em biblioteconomia (UFRGS).

E-mail: caterina@cpd.ufrgs.br.

\section{Janise Silva Borges da Costa}

Bibliotecária de sistemas, Centro de Processamento de Dados da UFRGS, bacharel em biblioteconomia (UFRGS).

E-mail: janise@cpd.ufrgs.br.

\section{Lais Freitas Caregnato}

Bibliotecária de sistemas, Centro de Processamento de Dados da UFRGS, especialista em automação de bibliotecas e centros de informações documentárias.

E-mail: lais@cpd.ufrgs.br.

Integrantes da Comissão de Automação do SBU.

\section{Resumo}

Utilização do conceito de controle bibliográfico definido pela IFLA e do formato MARC 21 bibliográfico para gerenciar a produção científica, técnica, artística e administrativa de uma instituição de ensino superior. Definição de campo MARC 9XX e de campos associados para registro das informações bibliográficas dos documentos de produção intelectual institucional. Contribuição da IES para o controle bibliográfico nacional.

\section{Palavras-chave}

Controle bibliográfico nacional; Controle bibliográfico institucional; Formato bibliográfico; Campo MARC 9XX; Produção intelectual; UFRGS.

The use of MARC 9XX field in institutional bibliographic control

\begin{abstract}
This paper describes the use of the concept of bibliographic control as defined by IFLA and of the MARC 21 bibliographic format to manage the scientific, technical, artistic and administrative production of a university-level institution. The definition of the MARC 9XX and associated fields for registering the bibliographic information of institutional intellectual production documents are examined besides the contribution of the institution for the national bibliographic control.
\end{abstract}

\section{Keywords}

National bibliographic control; Institutional bibliographic control; Bibliographic format; MARC 9XX field; Intellectual production; UFRGS.

\section{INTRODUÇÃO}

A invenção da imprensa e as quase ilimitadas possibilidades de edição eletrônica de documentos só fazem aumentar a angústia dos profissionais de informação, em face da necessidade de disciplinar a produção editorial, a fim de torná-la acessível ao maior número de indivíduos. O programa Controle Bibliográfico Universal (CBU) e o uso de formato de registro e intercâmbio de informações bibliográficas são propostas de alcance internacional para minimizar o problema de organização e uso sem barreiras da informação. A adoção desses conceitos em uma instituição de ensino superior contribui para reforçar o controle e a disseminação de sua produção intelectual, bem como para ampliar o número de entidades engajadas no controle bibliográfico nacional.

\section{CONTROLE BIBLIOGRÁFICO}

O inventário é uma memória auxiliar da mente humana. Um artifício criado pelo homem, a partir do momento em que o número de objetos a serem lembrados ou organizados tornou-se superior à sua capacidade de recuperar um item ou seu conteúdo.

Nos serviços de informação, o inventário transmutou-se em catálogo, para descrever e representar todos aqueles documentos armazenados em salas, estantes e arquivos e em bibliografias, elaborado com o intuito de controlar ou divulgar a produção bibliográfica em determinada área do conhecimento.

Do crescimento da produção editorial, gerando milhares de documentos em uma variedade de suportes, e da ampliação do fluxo de comunicação entre pessoas e instituições, decorreu a necessidade de codificar e organizar informações, para torná-las amplamente acessíveis. Buscando este controle bibliográfico mais eficiente, a Federação Internacional de Associações e Instituições Bibliotecárias (Ifla) e a Unesco lançaram, na década de 1970, o programa CBU, com "o objetivo de promover um sistema internacional para controle e intercâmbio de informações bibliográficas" (Anderson, 1974), tendo como elementos principais:

a disponibilidade universal de dados bibliográficos de todas as publicações de todos os países; em um 
formato de descrição internacionalmente aceito pelos participantes do programa (Campello \& Magalhães, 1997).

Para realizar seu controle bibliográfico, o país deve administrar a produção editorial identificando-a e registrando seus dados em conformidade com normas internacionais de descrição bibliográfica. Uma série de medidas, relacionadas com a origem, organização e normalização dos dados bibliográficos, deve ser adotada pelo país para gerir este controle (Kaltwasser, 1971):

1) estabelecer agência bibliográfica nacional, responsável, entre outras funções, pelo controle e identificação dos dados bibliográficos oficiais de cada publicação editada no país;

2) criar bibliografia nacional que arrole e divulgue, com regularidade e rapidez, os documentos publicados no país e editados pela agência;

3) criar corpo de legislação, o depósito legal, que defina quem e que tipo de documento está sujeito ao depósito legal, número de cópias e prazo de depósito e instituição depositária e responsável pelo registro/divulgação da produção editorial do país;

4) estabelecer a catalogação na publicação identificando os dados bibliográficos do documento que está sendo publicado, para divulgação em serviços específicos, facilitando os procedimentos de seleção e de aquisição de novos títulos;

5) buscar a padronização da descrição bibliográfica adotando padrões internacionais (AACR2, ISBD, MARC) para criação de registros bibliográficos que possam ser objeto de intercâmbio automatizado entre diferentes fontes e de interpretação independentemente do idioma do registro;

6) utilizar a identificação numérica do documento, atribuindo número padronizado (ISBN, ISSN) a cada título publicado no país para facilitar sua identificação, o controle editorial nacional e os processos de aquisição de documentos.

A magnitude da tarefa, considerando a diversidade e o volume da produção editorial de um país, pode tornar inviável este controle e divulgação por uma única agência bibliográfica nacional, aumentando a distância entre as propostas do CBU e o que a agência pode realizar. Para evitar o esvaziamento do programa, são propugnadas ações como iniciar o controle bibliográfico em esfera local (Campello \& Magalhães, 1997), a divisão das responsabilidades da agência com outras instituições, inclusive privadas, como editoras (Delsey, 1993) e sua gerência por indivíduos ou instituições especializadas, visando a atender interesses próprios de informação, diferentes dos demais usuários de um sistema, mas sem perder a perspectiva de integração com o controle bibliográfico nacional (Shera, 1975).

\section{FORMATO BIBLIOGRÁFICO}

O intercâmbio de informações bibliográficas entre instituições e países é elemento-chave no contexto proposto pelo CBU. Para que esta troca de informações se realize, é necessária uma linguagem comum aos participantes do programa. O formato bibliográfico, instrumento de racionalização de procedimentos na automação de serviços e produtos de informação, assume esta função de linguagem comum, a qual, ao disciplinar a descrição documental, viabiliza e otimiza o intercâmbio interinstitucional e internacional de informações bibliográficas.

O formato bibliográfico registra, segundo determinado padrão, as características bibliográficas do documento, gerando um registro (metadado) que substitui o item físico em um sistema de registro/recuperação de informações. Wellisch (1987) ressalta que o formato permite a completa descrição formal e física do documento, mas não a sua descrição temática, uma vez que esta depende do contexto onde o documento será utilizado. O Machine Readable Cataloging (MARC), ou registro legivel por computador, é um exemplo de formato de descrição bibliográfica, desenvolvido pela Library of Congress (LC) dos Estados Unidos, na década de 1960, quando iniciou a utilização do computador para gerenciar seu processo de catalogação. $O$ formato utiliza números, letras e sinais gráficos para marcar os campos e informações no registro bibliográfico (Furrie, 2003). O LC MARC evoluiu para o MARC 21 adotado internacionalmente como formato de registro bibliográfico pelas bibliotecas. O padrão proposto pelo LC MARC deu origem também a padrões MARC desenvolvidos em cada país, tais como UK MARC, no Reino Unido, CANMARC, no Canadá, e formatos CALCO e IBICT, no Brasil.

Enquanto a catalogação do documento utiliza as regras AACR2 para definir quais são e a forma das entradas e descrever fisicamente, o documento e as tabelas de classificação auxiliam na definição de seus assuntos, o formato MARC organiza essas informações de forma a serem lidas pelo computador e possibilita a descrição bibliográfica de diferentes tipos de documentos 
(monografia, arquivo de computador, música, material cartográfico e outros). O formato utiliza para tal a estrutura de campos fixos e variáveis, subcampos e indicadores.

Criado pela LC, a qual cumpre a função de uma agência bibliográfica nacional americana, para atender a suas necessidades de descrição bibliográfica, o MARC é um formato detalhado que oferece flexibilidade na seleção dos campos a serem utilizados para o registro de um documento, incluindo, ainda, um código para campos de uso local.

Os campos básicos do formato MARC para registro bibliográfico são:

- Líder - campo de 24 caracteres, em geral contendo informações para uso do próprio programa;

- Diretório - informa quais os campos utilizados e onde estão localizados no registro;

- 008 - campo de 40 caracteres contendo informações codificadas sobre o conteúdo do documento (tipo, país de publicação, público a que se destina, idioma etc.);

- Campos variáveis:

OXX - Informações de controle, números e códigos

1XX - Autoria (nome pessoal, entidade, evento)

2XX - Títulos, edição, imprenta

3XX - Descrição física

4XX - Série

$5 \mathrm{XX}-$ Notas

6XX - Entradas de assunto

7XX - Entradas secundárias (nome pessoal, entidade, evento, título)

8XX - Entradas secundárias de série

9XX - Uso local

Os campos 9XX e X9X foram criados para permitir o registro de informações de interesse específico para os usuários locais de um sistema de informação (Furrie, 2003) e não são incluídos quando o registro bibliográfico é objeto de intercâmbio.

\section{CONTROLE BIBLIOGRÁFICO INSTITUCIONAL}

A implantação de programa de controle bibliográfico em instituição de ensino superior amplia o controle, o uso e a divulgação da produção intelectual gerada por suas atividades de ensino, pesquisa e extensão. De forma mais específica, sua implantação possibilita:

- compartilhar a responsabilidade de controle de sua produção documental com a agência bibliográfica nacional, assumindo a tarefa em nível institucional;

- identificar a produção intelectual das suas diversas unidades acadêmicas e administrativas;

- apresentar um quadro de referência sobre a vocação e os interesses da instituição nas áreas de ensino, pesquisa e extensão;

- subsidiar processos de avaliação institucional que incluam variáveis de produção intelectual;

- fornecer cópias de documentos produzidos pela IES;

- divulgar a produção intelectual institucional;

- alimentar outros sistemas (p. ex. intercâmbio de dados com outras IES, com agência nacional bibliográfica, currículo Lattes de pesquisadores da instituição etc.) com registros bibliográficos oficiais, completos na descrição física do documento e indicadores de seu conteúdo temático básico;

- manter lista autorizada de nomes pessoais e de entidades coletivas pertencentes à instituição.

A implantação do controle bibliográfico institucional exige a adoção de medidas equivalentes às que gerenciam o controle bibliográfico nacional:

1) Depósito legal - edição de documentos normativos, como portarias da administração superior, que forneçam ordenamento ao processo de coleta da produção intelectual gerada nas diversas unidades e órgãos da instituição;

2) Agência bibliográfica - responsabilizar o serviço de informação bibliográfica (biblioteca central, serviço de documentação, sistema de bibliotecas) pelo controle e identificação dos dados bibliográficos oficiais de cada documento de produção intelectual gerado na instituição, pela guarda, fornecimento de cópias e divulgação dos mesmos;

3) Padronização da descrição bibliográfica - adotar padrões internacionais para criar registros bibliográficos que 
Zita Prates de Oliveira / Caterina Groposo Pavão / Janise Silva Borges da Costa / Lais Freitas Caregnato

identifiquem adequadamente a produção intelectual e possam ser objeto de intercâmbio com outras instituições.

Para criar registros bibliográficos completos de produção intelectual institucional, é necessário que a agência bibliográfica:

- defina fontes produtoras e tipos de produção intelectual, identificando as unidades acadêmicas e administrativas e os tipos de documentos por elas gerados e crie códigos para representar cada unidade e tipo;

- defina campo MARC 9XX no formato bibliográfico adotado pela IES para registro dos dados da produção institucional;

- crie índice específico no sistema de informações bibliográficas da IES para recuperação das informações codificadas de produção intelectual;

- crie relatórios que apresentem os registros de produção intelectual da IES por tipo de documento, por fonte produtora, por autor e outros de interesse institucional.

4) Bibliografia - implementar biblioteca digital a qual, cumprindo função de bibliografia institucional, registre e divulgue, com rapidez e de forma sistemática, o texto completo da documentação produzida e editada pela IES.

\section{Controle bibliográfico na UFRGS}

O controle bibliográfico da produção intelectual na UFRGS (PI) remonta aos anos 1970, quando foram realizados os primeiros esforços de automação de serviços bibliotecários na universidade.

Entre 1975 e 1983, fase inicial de automação do processo de catalogação no Sistema de Bibliotecas da UFRGS SBU, a coleta de PI restringiu-se a teses e dissertações. De 1984 a 1988, a automação das bibliotecas foi interrompida e não houve coleta/registro de PI. Somente a partir de 1989, com a implantação do Sistema de Automação de Bibliotecas (SABi), a coleta/registro de PI passou a ser realizada de forma contínua e sistemática na UFRGS.

A tabela 1 resume o trabalho de controle bibliográfico institucional realizado pelo SBU e alguns produtos editados pela universidade para divulgar sua produção intelectual.
Ao final de 2003, a base de dados de PI da UFRGS totalizava 107.438 registros bibliográficos de documentos produzidos pela instituição.

Em 2002, foi implantada a Biblioteca Digital de Teses e Dissertações para disponibilizar na Internet o acesso ao texto completo desses tipos de documentos produzidos na universidade, devendo ser ampliada para outros tipos de produção (material iconográfico, música, mapas, vídeo etc.) tão logo sejam concluídos os testes para sua operacionalização (Oliveira, 2003).

\section{Formato bibliográfico na UFRGS}

Adotando padrões internacionais que possibilitam o intercâmbio de informações bibliográficas com outras instituições, a UFRGS utiliza o AACR2 para determinar as entradas e os elementos de descrição dos documentos e o formato MARC 21 para registro dos dados em sua base bibliográfica.

Campos MARC adotados pelo formato bibliográfico definido pela UFRGS:

- Líder, Diretório e 008

- Campos variáveis: OXX (013, 020, 022, 024, 028, 030, 034, 040, 041, 043, 044, 045, 047, 048, 090, e 092)

- $\operatorname{1XX}(100,110,111$ e 130)

- $2 X X(210,222,240,242,245,246,250,254,255,260$ e 270)

- $3 X X(300,310,321$ e 362$)$

- $4 X X(440)$

- $5 \mathrm{XX}(500,501,504,505,508,510,511,515,518,520$, $525,530,538,546,550,555,561,580,581,590,591$ e 599)

Ci. Inf., Brasília, v. 33, n. 2, p. 179-186, maio/ago. 2004 
O uso do campo MARC 9XX para controle bibliográfico institucional

- 6 XX $(600,610,611,630,650,651,690$ e 692)

- $7 X X(700,710,711,730,740,760,762,765,767,770$, $772,773,775,777,780,785$ e 787$)$

- 8XX (856)

- 9XX (900, 901, 909, 910 e 930)

\section{Campo MARC 9XX na UFRGS}

Utilizando a estrutura de campo, subcampos e indicadores proposta pelo MARC, a Universidade criou o campo 909 para controle da produção intelectual. Foram também elaborados documentos auxiliares (glossário e tabelas de códigos) a serem utilizados no preenchimento do campo e definidos campos X9X e outros associados, visando a tornar mais completa a descrição bibliográfica e o acesso ao texto completo de um documento de produção intelectual na UFRGS.

\section{CAMPO 909 - PRODUÇÃO INTELECTUAL}

Campo optativo (OP); não repetitivo (NR).

Indicadores indefinidos.

Códigos de subcampo:

| a Unidade/Departamento/Órgão (OP, R)

|b Tipo de produção (OB, NR)

|c Curso de pós-graduação (OP, R)

|d Curso de especialização (OP, R)

| e Curso de graduação (OP, R)

|f Órgão financiador (OP, R)

Definição e conteúdo do campo

Campo utilizado para registro da produção científica, técnica, artística e administrativa da Instituição. Seu uso é obrigatório para documentos produzidos por integrantes do corpo docente, técnico-administrativo e discentes (desde que orientados por docente) da Universidade e para documentos produzidos pela UFRGS.

Indicadores

Indicadores 1 e 2 indefinidos, contêm branco ( ).

Códigos de subcampo

| a - Unidade/Departamento/Órgão
DES - Contém o código correspondente à unidade acadêmica ou administrativa da UFRGS produtora ou publicadora do documento, ou a qual o autor, orientador ou co-orientador está vinculado.

CAR - Subcampo optativo e repetitivo para documento e autores, orientadores, co-orientadores, produtores ou publicadores vinculados a diferentes unidades acadêmicas e administrativas da UFRGS.

NOT - Para preenchimento consulte a Tabela J Unidades/Departamentos/Órgãos da UFRGS.

| b - Tipo de produção intelectual

DES - Contém o código correspondente ao tipo de produção contida no documento. Em caso de dúvida quanto ao tipo de produção, consulte o Anexo 2. Conceituação dos tipos de produção intelectual.

CAR - Subcampo obrigatório, não repetitivo.

NOT - Para preenchimento deste subcampo, use os códigos abaixo:

Apostila - pc

Arquivo de computador - pi

Artigo de divulgação - pp

Artigo publicado em periódico indexado estrangeiro pfi

Artigo publicado em periódico indexado nacional - pgi

Artigo publicado em periódico não indexado estrangeiro - pfn

Artigo publicado em periódico não indexado nacional - pgn

Capítulo de livro - pb

Catálogo de cursos de graduação, pós-graduação, extensão etc. - pr

Catálogo de evento - ps

Dissertação - pe

Documento jurídico - pq

Entrevista - pab

Livro - pa

Material cartográfico - pk 
Material visual - px

Música - pv

Outros documentos $-\mathrm{pz}$

Palestra - pac

Patente - pu

Projeto arquitetônico - par

Projeto paisagístico - pas

Projeto urbanístico - pau

Publicação seriada - pt

Relatório administrativo - pm

Relatório técnico e de pesquisa - pl

Tese - pd

Texto de apresentação - pta

Trabalho de conclusão de curso de mestrado profissional - pep

Trabalho de conclusão de disciplina/curso de ensino fundamental e médio - pef

Trabalho de conclusão de disciplina/curso de ensino profissional - per

Trabalho de conclusão de disciplina/curso de especialização - pn

Trabalho de conclusão de disciplina/curso de graduação - po

Trabalho de conclusão de disciplina de doutorado - ped

Trabalho de conclusão de disciplina de mestrado - pem

Trabalho de conclusão de disciplina de mestrado profissional - peo

Trabalho publicado em anais de evento realizado fora do país - pj

Trabalho publicado em anais de evento realizado no país - ph

Trabalho técnico - pad

Tradução - paf |c - Curso de pós-graduação

DES - Contém o código correspondente ao curso de pós-graduação da UFRGS produtor ou publicador do documento, ou ao qual o autor, orientador ou coorientador está vinculado.

CAR - Subcampo optativo e repetitivo para documento e autores, orientadores, co-orientadores, produtores ou publicadores vinculados a diferentes cursos de pósgraduação da UFRGS.

NOT - Para preenchimento deste subcampo, consulte a Tabela K - Cursos de Pós-graduação da UFRGS.

|d - Curso de especialização

DES - Contém o código correspondente ao curso de especialização da UFRGS produtor ou publicador do documento ou ao qual o autor, orientador ou coorientador está vinculado.

CAR - Subcampo optativo e repetitivo para documento e autores, orientadores, co-orientadores, produtores ou publicadores vinculados a diferentes cursos de especialização da UFRGS.

NOT - Para preenchimento deste subcampo, consulte a Tabela M - Cursos de Especialização da UFRGS.

| e - Curso de graduação

DES - Contém o código correspondente ao curso de graduação da UFRGS produtor ou publicador do documento ou ao qual o autor, orientador ou coorientador está vinculado.

CAR - Subcampo optativo e repetitivo para documento e autores, orientadores, co-orientadores, produtores ou publicadores vinculados a diferentes cursos de graduação da UFRGS.

NOT - Para preenchimento deste subcampo, consulte a Tabela L - Cursos de Graduação da UFRGS.

|f - Órgão financiador

DES - Contém o nome ou sigla do órgão financiador, como, por exemplo, CNPq, Finep e Fapergs.

CAR - Subcampo optativo e repetitivo para documentos que contêm menção ao órgão financiador. 


\section{Exemplos}

FIGURA 1

Registro de tese defendida no Programa de Pós-graduação em Engenharia de Minas, Metalúrgica e de Materiais da Escola de Engenharia da UFRGS

\begin{tabular}{|c|c|c|c|c|}
\hline Líder & LDR & 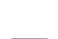 & 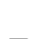 & $----n t m--22----4 a-4500$ \\
\hline Campo controle & 008 & $\longrightarrow$ & - & -----s2003----bl-ado---m---f000-0-por-d \\
\hline Fonte catalog. & $\overline{040}$ & 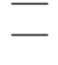 & $\overline{\bar{a}}$ & $\begin{array}{l}\text { BIPaURS } \\
\text { por }\end{array}$ \\
\hline Idioma & 041 & 0 & $\bar{a}$ & nor \\
\hline Título & 245 & 10 & $\frac{\mathrm{a}}{\mathrm{b}}$ & $\begin{array}{l}\text { Introdução de amostras sólidas em espectrometria de absorcão atômica } \\
\text { com chama : } \\
\text { determinação de cobre, chumbo, zinco e ouro em amostras geológicas } \\
\text { José Neri Gottfried Paniz }\end{array}$ \\
\hline Área public. & 260 & & $\bar{c}$ & 2003. \\
\hline Desc. fisica & 300 & 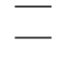 & $\frac{\bar{a}}{b}$ & $\begin{array}{l}x x, 85 \mathrm{f} . \\
\text { il. }\end{array}$ \\
\hline Nota tese/diss. & 591 & $\underline{0}$ & $\bar{a}$ & Tese (doutorado) \\
\hline & & & $\frac{\bar{b}}{\mathrm{c}}$ & $\begin{array}{l}\text { Universidade Federal do Rio Grande do Sul. Escola de Engenharia. } \\
\text { Programa de Pós-Graduação em Engenharia de Minas, Metalúrgica e de } \\
\text { Materiais } \\
2003 \text {. } \\
\text { Porto Alegre, BR-RS }\end{array}$ \\
\hline Assunto & 650 & 04 & $\bar{a}$ & Espectrometria de absorção atômica \\
\hline Assunto & $\overline{650}$ & $\overline{04}$ & $\bar{a}$ & Decomposição de amostras \\
\hline Assunto & $\overline{650}$ & $\overline{04}$ & $\frac{\bar{a}}{x}$ & $\begin{array}{l}\text { Elementos-traço } \\
\text { Determinação }\end{array}$ \\
\hline Macrodescritor & 690 & & $\bar{a}$ & Engenharia metalúrgica \\
\hline Macrodescritor & 690 & & $\bar{a}$ & Química analítica \\
\hline Orientação & 900 & 0 & $\bar{a}$ & Martins, Ayrton Figueiredo \\
\hline Prod. intelec. & 909 & 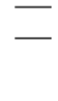 & $\frac{\bar{a}}{\mathrm{~b}}$ & $\begin{array}{l}\text { ENGO } \\
\text { pd } \\
\text { METP }\end{array}$ \\
\hline Dados da bib. & 910 & & $\bar{a}$ & ENG \\
\hline
\end{tabular}

\section{FIGURA 2}

Registro de artigo de periódico nacional que caracteriza produção intelectual do Departamento de Odontologia Conservadora da Faculdade de Odontologia da UFRGS

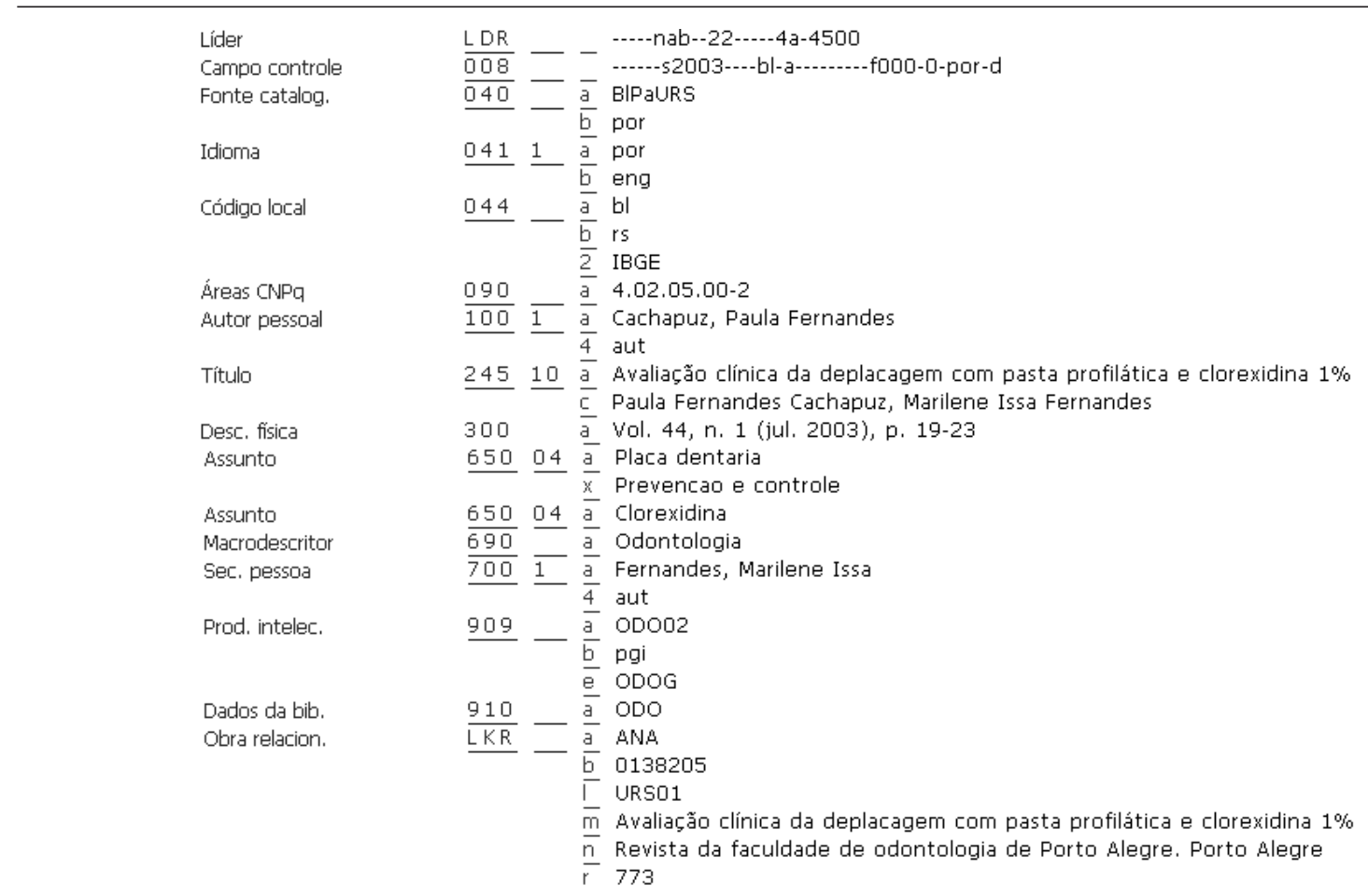




\section{INSTRUMENTOS AUXILIARES AO PREENCHIMENTO} DO CAMPO 909

a) Manual de Registro Bibliográfico,

Anexo 2 - Conceituação dos Tipos de Produção Intelectual, para definições e esclarecimentos sobre cada tipo de produção.

b) Tabelas Auxiliares

- Tabela J - Unidades/Departamentos/Órgãos da UFRGS lista códigos para cada unidade, departamento e órgão da Universidade

- Tabela K - Cursos de Pós-Graduação da UFRGS lista códigos para cada curso de pós-graduação da Universidade

- Tabela L - Cursos de Graduação da UFRGS lista códigos para cada curso de especialização da Universidade

- Tabela M - Cursos de Especialização da UFRGS lista códigos para cada curso de especialização da Universidade

c) Manual de Políticas \& Procedimentos de Processamento Técnico

- Capítulo 2 - Diretrizes para Registro de Produção Intelectual consolida orientações emanadas do SBU para o registro de PI na base de dados SABi.

\section{CAMPOS ASSOCIADOS}

090 - Classificação por áreas de conhecimento do CNPq

591 - Nota de tese ou dissertação

856 - Localização e acesso eletrônico

900 - Orientação de trabalho acadêmico

\section{CONSIDERAC̣ÕES FINAIS}

A criação de um campo para controle bibliográfico da produção intelectual da universidade foi uma aposta estratégica da Direção da Biblioteca Central nos anos 1980 para fortalecer e consolidar o sistema de automação de bibliotecas junto à Administração Central da UFRGS. A partir do registro sistemático de sua produção intelectual pelo sistema de bibliotecas, a universidade passou a contar com dados valiosos para processos de avaliação institucional e para divulgação de suas atividades de ensino, pesquisa e extensão.

A contribuição da UFRGS para o controle bibliográfico nacional não se limita à coleta, registro e disponibilidade de sua produção intelectual. Também se inclui neste procedimento o trabalho criterioso de definição de entradas de nomes pessoais e institucionais e a sua consistência na base de dados para documentos de PI, fixando estas entradas como um catálogo de autoridades para nomes oriundos da universidade.

O conceito de controle bibliográfico proposto pela Ifla na década de 1970 permanece atual e, associado a tecnologias de informação como bancos de dados e ao uso de formato de descrição documental, possibilita a uma instituição aprimorar o controle de sua produção intelectual e integrar-se aos esforços do controle bibliográfico nacional.

Artigo recebido em 12-06-2004 e aceito para publicação de 18 a 21/10/2004.

\section{REFERENCIAS}

ANDERSON, Dorothy. Universal bibliographic control: a long term policy, a plan for action. München : Verlag Documentation, 1974.

CAMPEllo, Bernadete Santos; MAGAlHÃes, Maria Helena de Andrade. Introdução ao controle bibliográfico. Brasília : Briquet de Lemos Livros, 1997.

CONSELHO NACIONAL DE DESENVOLVIMENTO CIENTÍFICO E TECNOLÓGICO. Currículo lattes. Disponível em: <http:// www.cnpq.br/plataformalattes>. Acesso em: 13 jan. 2004.

DELSEY, Tom. Universal bibliographic control in a changing context. In: IFLA SEMINAR ON UNIVERSAL BIBLIOGRAPHIC CONTROL, 1993, Rio de Janeiro. Papers... [ S.1.: s.n.], 1993. p. 16-41.

FURRIE, Betty. Understanding MARC bibliographic. Disponível em: <http://www.loc.gov/marc/umb/um01to06.html>. Acesso em: 24 dez. 2003.

KARLTWASSER, Franz Geog. El control bibliográfico universal. Boletin de la Unesco para las Bibliotecas, v. 25, n. 5, p. 269-277, 1971.

MARC 21: formato condensado para dados bibliográficos. Marília, SP : UNESP, Marília Publicações, 2000. 2 v.

MEY, Eliane Serrão Alves. Considerações (preguiçosas) sobre a prática da catalogação. Revista de Biblioteconomia de Brasília, Brasília, v. 19, n. 2, p. 127-136, jul./dez. 1995.

- Não brigue com a catalogação. Brasília : Briquet de Lemos Livros, 2003.

OLIVEIRA, Zita Prates de et al. Implementação da biblioteca digital da UFRGS. Renote: revista novas tecnologias na educação, Porto Alegre, set. 2003. Disponível em <http://www.cinted.ufrgs.br/renote/ set2003/artigos/implementacaobiblioteca.pdf>. Acesso em: 8 mar. 2004.

SHERA, Jesse H. Bibliographic management. In: BRENNY, Vito. Essays on bibliography. Metuchen : Scarecrow, 1975. p. 167-175.

UNIVERSIDADE FEDERAL DO RIO GRANDE DO SUL. Biblioteca Central. SABi: manual de registro bibliográfico. Porto Alegre, 2004.

WELLISH, Hans. A cibernética do controle bibliográfico: para uma teoria dos sistemas de recuperação da informação. Brasília : IBICT, 1987. 